\title{
Classical conditioning of attitudes as a function of persuasion trials and source consensus
}

\author{
ROBERT FRANK WEISS and MICHELE K. STEIGLEDER \\ University of Oklahoma, Norman, Oklahoma 73019 \\ RICHARD A. FEINBERG \\ Department of Consumer Sciences, Purdue University, West Lafayette, Indiana 47907 \\ and \\ ROBERT ERVIN CRAMER \\ California State College, San Bernardino, California 92407

\begin{abstract}
Conditioned attitude strength was an increasing function of number of persuasion trials and source consensus, and these two variables interacted so that the effects of source consensus became more pronounced as the number of persuasion trials increased. Results were predicted from a theory of persuasive communication in which persuasion trials are analogous to conditioning trials, source consensus is analogous to UCS strength, and probability of agreement is analogous to $\mathrm{CR}$ probability.
\end{abstract}

Persuasion and the acquisition of attitudes have enjoyed some illumination from the application of instrumental and classical conditioning models (e.g., Weiss, 1968; Weiss, Miller, Langan, \& Cecil, 1971; Weiss, Wenninger, Balling, \& Miller, 1980). This report further explores the possibilities of a classical conditioning model in which the opinion to be learned is the conclusion of a syllogism. Subjects are exposed to a persuasive communication that includes the premises of the syllogism but not the conclusion (McGuire, 1960, 1981; Wyer \& Hartwick, 1980), and the subject is left to draw the implied conclusion himself (Hovland $\&$ Mandell, 1952). Figure 1 shows corresponding paradigms for classical conditioning and attitude learning. Research based on this classical conditioning model requires persuasive communications that incorporate two elements: (1) the opinion-eliciting argument (syllogism premises), and (2) the cue statement, two (neutral) words that immediately precede the opinioneliciting argument and will later constitute part of the test used to measure attitude acquisition. The cue statement precedes the opinion-eliciting argument, so that a subject listening to (or reading) the communication will first hear the cue statement, followed by the opinion-eliciting argument (syllogism premises), and then draw the conclusion implied by the argument. This sequence of events may be regarded as analogous to the sequence: CS, UCS, UR. The cue statement is the CS, and the opinion-eliciting argument is the UCS, which elicits the implied opinion: the UR. Through repetition of the sequence, the implied opinion (UR) becomes

Reprints may be obtained from R. F. Weiss.
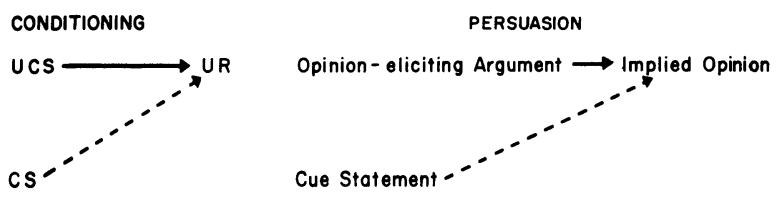

Figure 1. Corresponding paradigms for a classical conditioning trial and a persuasion trial.

conditioned to the cue statement (CS) and thus becomes a conditioned opinion (CR), as shown in Figure 1.

Following the logic of this paradigm further, the number of repetitions of this sequence is analogous to the number of conditioning trials and the power of the opinion-eliciting argument to convincingly imply the opinion is analogous to the strength of the UCS. The argument is attributed to a group source (experts, eyewitnesses) that is varied only in the degree of consensus among these experts or eyewitnesses. The degree of consensus among the sources of the argument affects the power of the argument to convincingly imply the opinion and, hence, also corresponds to UCS strength. Research in classical conditioning indicates that CR strength (as measured by response probability) is an increasing function of trials and UCS strength, and that these two variables typically interact so that the effects of UCS strength are more pronounced as the number of trials increases. Similarly, our theory predicts that attitude strength (as measured by response probability) should be an increasing function of persuasion trials and source consensus, and these two variables should interact so that the effects of source consensus should be more pronounced as the number of persuasion trials increases. 


\section{METHOD}

The subjects were 169 undergraduates. The design was a 2 by 2 factorial with 34 subjects in each of the four cells. There were two levels of number of persuasion trials (one vs. three readings of the persuasive communications) and two levels of consensus among the sources of the argument. There was also a no-persuasion control group $(\mathrm{N}=33)$, which was exposed to the same buffer communications as the experimental groups but not to the persuasive communications.

Under the impression that he was participating in a study of "Speech Patterns and Decision-Making," each experimental subject read three persuasive communications and three buffers into an "electronic speech analyzer." One-trial subjects read each communication once, and three-trial subjects read each communication three times. There were three reading sessions within the $35-$ to $45-\mathrm{min}$ experimental period, each followed immediately by attitude measurement in order to minimize any possible sleeper effects (e.g., Cook, Gruder, Hennigan, \& Flay, 1979). Each reading session covered one persuasive communication and one buffer.

In order to study "conditioning" rather than "habit reversal," the persuasive communications were directed at fictitious opinion topics, on which $95 \%-98 \%$ of a sample of 100 similar undergraduates had no opinion (e.g., the Kopaks are descended from the ancient Mongols). The high- and low-consensus values were (1) all vs. a minority of the scientists, (2) 182 vs. 51 , of 202 , eyewitnesses to the plane crash, and (3) all vs. $25 \%$ of the linguistic experts.

The attitude measuring apparatus assessed each subject's probability of agreement with the opinion after the subject had been exposed to persuasive communication. The cue statement and opinion were projected on a screen (preceded by six to seven buffers), and the subject signified his agreement (if he agreed) by moving a lever toward the opinion. The subject was allowed $40 \mathrm{sec}$ in which to respond. Subjects who did not agree did not move the lever. Probability of agreement was calculated for each subject from the data of the three test trials. The persuasion and measurement procedures are analogous to conditioning procedure, in some detail (Weiss, 1968).

\section{RESULTS}

Source consensus and persuasion trials interacted so that the effects of source consensus became more pronounced as the number of persuasion trials increased $[F(1,132)=9.54, p<.01]$. Probability of agreement was an increasing function of source consensus $[F(1,132)=42.92, p<.01]$. Probability of agreement increased from one to three trials only for the highconsensus groups $[\mathrm{t}(66)=2.32, \mathrm{p}<.05]$, although probability did increase radically from zero trials (control) to one trial of low consensus, showing clearly that the low-consensus communication was effective. The low-consensus group decreased, nonsignificantly, in probability of agreement from one to three trials, so that after three low-consensus trials, attitude strength remained far above the controls.

\section{DISCUSSION}

The research reported here derives from a theory of attitude learning (Weiss, 1968) that is based on systematic analogies with learning research and neo-Hullian learning theory. The theory was formulated in sufficient detail to permit derivations that are not always intuitively obvious, such as interaction effects and curve shapes. Thus the theory predicted not only the increase in attitude strength as a function of the trials and consensus variables, but also the interaction between these variables. These results confirm a previous consensus-by-trials experiment that compared one and two persuasion trials rather than the present one and three (Weiss, 1968). The results of another study also support the theory: As in classical conditioning, probability of agreement was a negatively accelerated increasing function of source consensus (Weiss, 1968). The results reported here closely resemble those obtained for another analog of UCS strength, argument strength, in interaction with number of persuasion trials (Weiss, 1968).

\section{REFERENCES}

Cook, T. D., Gruder, C. L., Hennigan, K. M., \& Flay, B. R. History of the sleeper effect: Some logical pitfalls in accepting the null hypothesis. Psychological Bulletin, 1979, 86, 662-679.

HovLAND, C. I., \& MANDELL, W. An experimental comparison of conclusion-drawing by the communicator and by the audience. Journal of Abnormal and Social Psychology, 1952, 47, 581588.

McGuIre, W. J. A syllogistic analysis of cognitive relationships. In M. J. Rosenberg, C. I. Hovland, W. J. McGuire, R. P. Abelson, \& J. W. Brehm, Attitude organization and change. New Haven, Conn: Yale University Press, 1960.

McGuIRE, W. J. The probabilogical model of cognitive structure and attitude change. In R. E. Petty, T. M. Ostrom, \& T. C. Brock (Eds.), Cognitive responses in persuasion. Hillsdale, N. J: Erlbaum, 1981.

WE iss, R. F. An extension of Hullian learning theory to persuasive communication. In A. G. Greenwald, T. C. Brock, \& T. M. Ostrom (Eds.), Psychological foundations of attitudes. New York: Academic Press, 1968.

Weiss, R. F., Miller, F. G., Langan, C. J., \& Cecil, J. S. Social facilitation of attitude change. Psychonomic Science, 1971, 22, 113-114.

Weiss, R. F., Wenninger, V. L., Ballina, S. S., \& Miller, F. G. Monotonicity of drive effects in the instrumental conditioning of attitudes. Bulletin of the Psychonomic Society, 1980, 16, 381-382.

WYER, R. S., JR., \& HARTwick, J. The role of information retrieval and conditional inference processes in belief formation and change. In L. Berkowitz (Ed.), Advances in experimental social psychology (Vol. 13). New York: Academic Press, 1980.

(Received for publication May 26, 1982.) 